\title{
Intrasaccadic Perception
}

\author{
Miguel A. García-Pérez ${ }^{1}$ and Eli Peli ${ }^{2}$ \\ ${ }^{1}$ Departamento de Metodología, Facultad de Psicología, Universidad Complutense, 28223 Madrid, Spain, and ${ }^{2} T h e$ \\ Schepens Eye Research Institute, Harvard Medical School, Boston, Massachusetts 02114
}

\begin{abstract}
Mammalian vision has a lowpass frequency characteristic that filters out fast temporal oscillations. Thus, fast-drifting gratings cannot be detected with static eyes, but the same gratings can easily be detected by executing saccades. Because these gratings are invisible under fixation, they are useful for isolating and studying intrasaccadic perception, which is normally masked by presaccadic and postsaccadic perception. We have conducted a number of psychophysical studies using these stimuli, and here we report that intrasaccadic visual processing allows for motion perception, that gratings drifting in the direction of a saccade are perceived as having more contrast than the same gratings drifting in the opposite direction, and that
\end{abstract}

intrasaccadic contrast perception has sufficient grain to allow psychophysical matching of the perceived contrast of gratings drifting in opposite directions. The conditions in which these phenomena occur disprove a recent hypothesis that intrasaccadic motion perception occurs for stimuli processed by the magnocellular system, and our results can be explained by assuming that the temporal lowpass characteristic that accounts for flicker fusion phenomena under vision with static eyes is also operative during saccades.

Key words: saccades; saccadic suppression; intrasaccadic perception; image motion; human; temporal impulse response
Vision in natural conditions requires eye movements, but these pose substantial problems for vision research. In psychophysical research, saccadic eye movements allow the detection of stimuli that move at such a fast speed that they are undetectable under fixation (Kelly, 1972, 1990; Deubel et al., 1987; Castet and Masson, 2000). This phenomenon has been attributed to the fact that eye movements lower the retinal velocity of the stimulus, bringing it to a visible range. The explanation is supported by evidence that sensitivity during pursuit of drifting gratings equals sensitivity to static gratings under fixation (Murphy, 1978; Flipse et al., 1988; Peli et al., 1998). Thus, sensitivity is governed by retinal motion, not by motion within an external reference system.

The fact that saccades bring into visibility what otherwise are undetectable fast-moving stimuli raises a theoretical question. Extensive research has established that contrast thresholds during saccades are often higher than thresholds under fixation, a phenomenon that has given rise to the concept of saccadic suppression (Matin, 1974; Volkmann, 1986). It has further been shown (Burr et al., 1994) that saccadic suppression selectively affects the magnocellular visual pathway: sensitivity decreases during saccades only for stimuli processed through this pathway (low spatial frequencies and high temporal frequencies). This selective degradation of visual processing has been assumed to serve the goal that the world remains stable despite considerable motion induced by saccades (Burr et al., 1994). Yet, the fact that fast-moving gratings of low spatial frequency that are invisible

Received Jan. 22, 2001; revised June 26, 2001; accepted June 26, 2001.

This work was performed at The Schepens Eye Research Institute, where M.A.G.-P. was a Research to Prevent Blindness International Research Scholar. This work was also supported by Dirección General de Enseñanza Superior Grant PB96-0597 and by a Schepens Eye Res Institute Career Enhancement grant to E.P. E.P. was supported by National Institute of Health Grants EY05957 and EY12890 and by NASA Grant NCC-2-1039.

Correspondence should be addressed to Dr. Miguel A. García-Pérez, Departamento de Metodología, Facultad de Psicología, Universidad Complutense, Campus de Somosaguas, 28223 Madrid, Spain. E-mail: miguel@psi.ucm.es.

Copyright (C) 2001 Society for Neuroscience $\quad 0270-6474 / 01 / 217313-10 \$ 15.00 / 0$ under fixation become visible during saccades suggests that saccadic suppression does not eliminate the perception of highcontrast stimuli. Because vision in natural conditions usually involves high contrasts, an investigation into the characteristics of intrasaccadic visual processing seems necessary.

The effect of saccades on vision has traditionally been studied with stimuli that are visible under fixation, because only then can sensitivity with and without saccades be compared. One problem with this approach is that it speaks of intrasaccadic processing only indirectly by way of a deterioration with respect to performance under fixation. This approach cannot pinpoint the cause of the deterioration, nor can it determine what visual processes are affected and what others remain functional.

To overcome these difficulties, here we investigate intrasaccadic processing directly using stimuli that are invisible under fixation and whose perception must occur during saccades. With these stimuli, intrasaccadic perception is effectively isolated for study. Our results indicate that intrasaccadic visual processing allows performing a number of complex visual tasks such as directionof-motion discrimination, contrast discrimination, and contrast matching. Because we used stimuli that are processed through the magnocellular pathway, our results further indicate that saccadic suppression does not cancel the processing of high-contrast stimuli during saccades and, therefore, that saccade-induced retinal motion must be compensated for by some other mechanism for the world to remain stable during saccades.

\section{MATERIALS AND METHODS}

Viewing was binocular with natural pupils and accommodation. All experiments were self-paced. If the subjects had blinked, had failed to maintain steady fixation, or had failed to execute the required saccade (as appropriate), the trial was discarded. All subjects (one of the authors and three naive subjects with normal or corrected-to-normal vision) were experienced observers.

Sampled display and stimuli. An EIZO FlexScan FX-E7 21 inch monitor was used at a frame rate of $122.6 \mathrm{~Hz}$. The monitor was linearized by gamma correction. Mean luminance was $34 \mathrm{~cd} / \mathrm{m}^{2}$. All experimental 
events were controlled by VisionWorks (Swift et al., 1997). Stimuli were Gabor patches with a static circular Gaussian aperture and a drifting carrier. Spatial frequencies were $0.2,0.5$, and $1 \mathrm{cycle} /{ }^{\circ}$, and temporal frequency varied up to $61.3 \mathrm{~Hz}$. Aperture size varied across experiments. Stimuli were presented for $1000 \mathrm{msec}(500 \mathrm{msec}$ in the experiment requiring fixation) with linearly ramped onsets and offsets (125 $\mathrm{msec}$ each). Except where otherwise indicated, contrast was $80 \%$ for the 0.2 cycle $/{ }^{\circ}$ patches and $50 \%$ for the 0.5 and 1 cycle $/{ }^{\circ}$ patches. Viewing distance was $65 \mathrm{~cm}$.

Continuous display and stimuli. The apparatus consisted of a wheel whose circumference presented a locally flat surface $174 \mathrm{~cm}$ around and $3 \mathrm{~cm}$ wide. Rotation of the wheel was achieved by a DC motor rendering speeds between 43 and $243 \mathrm{rpm}$. Square wave gratings of 0.2 and 1 cycle/cm were laser printed at 600 dots per inch, and the printouts were mounted around the wheel. The apparatus was hidden from view by a board with an opening $8 \times 2 \mathrm{~cm}$. Marks on the board immediately below the viewing aperture served as guides for saccades. From the viewing distance of $57 \mathrm{~cm}$, rotation at $1 \mathrm{rpm}$ produces an almost flat stimulus drifting at $2.9 \%$ sec. A DC light source illuminated the front of the apparatus.

Control of eye movements. Subjects were tested for accurate fixation and appropriate saccades. They performed the experimental tasks while their eye movements were recorded with an infrared corneal-reflection eye-tracking system (ISCAN Inc., Burlington, MA). Off-line analyses indicated that all subjects maintained fixation when required and that they executed appropriate 2 and $10^{\circ}$ saccades. In early recordings during fixation, subjects occasionally reported a flash-like appearance of the gratings with microsaccades that were impossible to tell from others that did not elicit this appearance. Given the impossibility of discarding inappropriate data by analysis of eye-movement patterns, subjects were warned that the stimuli in the experiment requiring fixation had a long presentation duration and, then, that they should discard trials in which stimulus duration appeared to be much briefer. None of the subjects reported any difficulty in identifying the trials in which this had occurred. All other experiments involved saccades and did not require such action on the part of the subjects.

Visibility elicited by saccades: sampled display. Stimuli $\left(9 \times 9^{\circ}\right)$ consisted of gratings of 0.2 and 1 cycle $/{ }^{\circ}$ in apertures with space constants of $3^{\circ}$. In the experiment requiring fixation, carriers were oriented vertically and drifted to the right. In the experiment involving saccades, carriers were vertical drifting rightward, vertical drifting leftward, and horizontal drifting downward. A cross (luminance, $41 \mathrm{~cd} / \mathrm{m}^{2}$; arm length, $0.2^{\circ}$ ) served as a fixation aid in the experiment requiring fixation. In the experiment involving saccades, two dots (luminance, $3 \mathrm{~cd} / \mathrm{m}^{2}$; radius, $0.1^{\circ}$ ) were aligned horizontally and centered on the image area of the monitor, either 2 or $10^{\circ}$ apart from one another to induce horizontal saccades. A spatial two-alternative forced-choice (2AFC) detection task was used with the method of constant stimuli, and the stimulus appeared either directly above or below the center of the monitor (at random on each trial). In the central (but not foveal) fixation condition, the fixation mark was on the center of the monitor; in the peripheral condition, it was $5^{\circ}$ to the left of that position. The subject responded whether the stimulus had appeared in the upper or the lower location. In the experiment involving saccades, each trial started while the subject was fixating on the mark on the left. One third of the time into the presentation interval, this mark was removed, and the mark on the right presented, directing the subject to execute a saccade. The subject responded whether the stimulus had appeared above or below the saccade path.

Visibility elicited by saccades: continuous display. Using a modified method of constant stimuli, the experimenter decided on the number of trials at each velocity, which varied between 10 and 40 depending on the subject's performance. Order of presentation was reasonably random. On each trial, the wheel was first set in motion with the opening blocked, then the subject was asked to fixate on the mark on the left of the viewing aperture, and the opening was exposed. The subject was asked to execute a saccade to the fixation mark on the right, pause briefly, and return with a saccade in the reverse direction. Subjects indicated which saccade direction made the stimulus visible. Saccades had amplitudes of 2, 10, or $20^{\circ}$. Auditory masking prevented frictional noise-which was directly related to speed-from providing contaminating clues.

Intrasaccadic motion perception. Stimuli $\left(11 \times 3^{\circ}\right)$ consisted of vertical gratings of $0.2,0.5$, and 1 cycle $/^{\circ}$. A spatial $2 \mathrm{AFC}$ task was used with the method of constant stimuli. A grating drifting in one direction (at random on each trial) was displayed directly above and vertically centered with the saccade path while the same grating but drifting in the opposite direction was displayed directly below the saccade path. Saccades were induced as described earlier. Subjects responded whether the rightward-drifting grating was above or below the saccade path, and they were asked to respond either by direct perception of the rightwarddrifting grating or by elimination after perception of the leftward-drifting grating. Subjects were instructed to ignore differences other than in direction of motion. To prevent subjects from using a guessing strategy that might contaminate the results, they were instructed to use always the same response key when they could not see motion. This strategy does not bias the results because target location was randomized.

Direction-related differences in perceived contrast. This experiment used the same stimulus set and procedure as the preceding one, but subjects indicated which stimulus had higher contrast.

Contrast-matching of stimuli drifting in opposite directions. Trials had the same design as in the preceding experiment. Subjects indicated whether the contrast of the grating in the upper position was higher than that of the grating underneath. Data were collected with an adaptive method of constant stimuli governed by two interwoven, 40-reversal, up-down staircases with step sizes of $0.05 \log$ units. Trial responses were aggregated and binned by contrast level to obtain percentage points, and a logistic function was fitted by maximum-likelihood methods.

\section{RESULTS \\ Visibility elicited by saccades}

A preliminary experiment showed that when grating speed is such that temporal frequency is above $\sim 45 \mathrm{~Hz}$, the gratings cannot be detected under fixation, neither foveally nor peripherally (Fig. $1 a)$. However, when the subjects execute saccades during the presentation period, the gratings are detected almost always (Fig. $1 b)$, regardless of the amplitude of the saccade $\left(2\right.$ or $\left.10^{\circ}\right)$ and also regardless of the direction of the saccade with respect to the direction of stimulus motion (along, against, or orthogonal). Because the gratings were not detected peripherally under fixation (Fig. 1a), their detection is not a result of their falling onto the motion-sensitive peripheral visual field (Kelly, 1984) before or after saccades. The gratings were definitely detected during the saccade.

Our results under fixation agree with extensive data on the temporal-frequency cutoff of vision (Robson, 1966; van Ness et al., 1967; Kulikowski, 1971; Kelly, 1979; Koenderink and van Doorn, 1979; Burr and Ross, 1982; Watson et al., 1986), and our results during saccades can be interpreted with reference to the peculiarities of motion induced by saccades. (A formal analysis is presented in the Appendix.) The fact that all gratings are visible with saccades along their direction of motion (Fig. $1 b$, left column) agrees with the principle that these saccades reduce retinal velocity, bringing energy into the window of visibility (Fig. 2a). But stimuli are also visible with saccades against their direction of motion (Fig. 1b, center column) although, by the same principle, these saccades increase retinal velocity and, then, they should further hinder detection. Yet, because cathode-ray tubes (CRTs) are time-sampled devices, drifting stimuli displayed on them have always temporal-frequency replicas that are usually outside the limits of the window of visibility. These replicas are responsible for the spread of energy into the window of visibility, thus helping detection with saccades against the direction of motion of the stimulus (Fig. 2b).

On the other hand, orthogonal saccades also help detect the stimuli (Fig. 1b, right column), although these saccades do not alter the retinal velocity of the gratings. Yet, horizontal eye movements occasionally include some vertical motion, often at the end and to compensate for vertical drift along the horizontal displacement (Rottach et al., 1998). This vertical component is sufficient to spread energy into the window of visibility in a manner that is consistent with our results. For a given temporal 


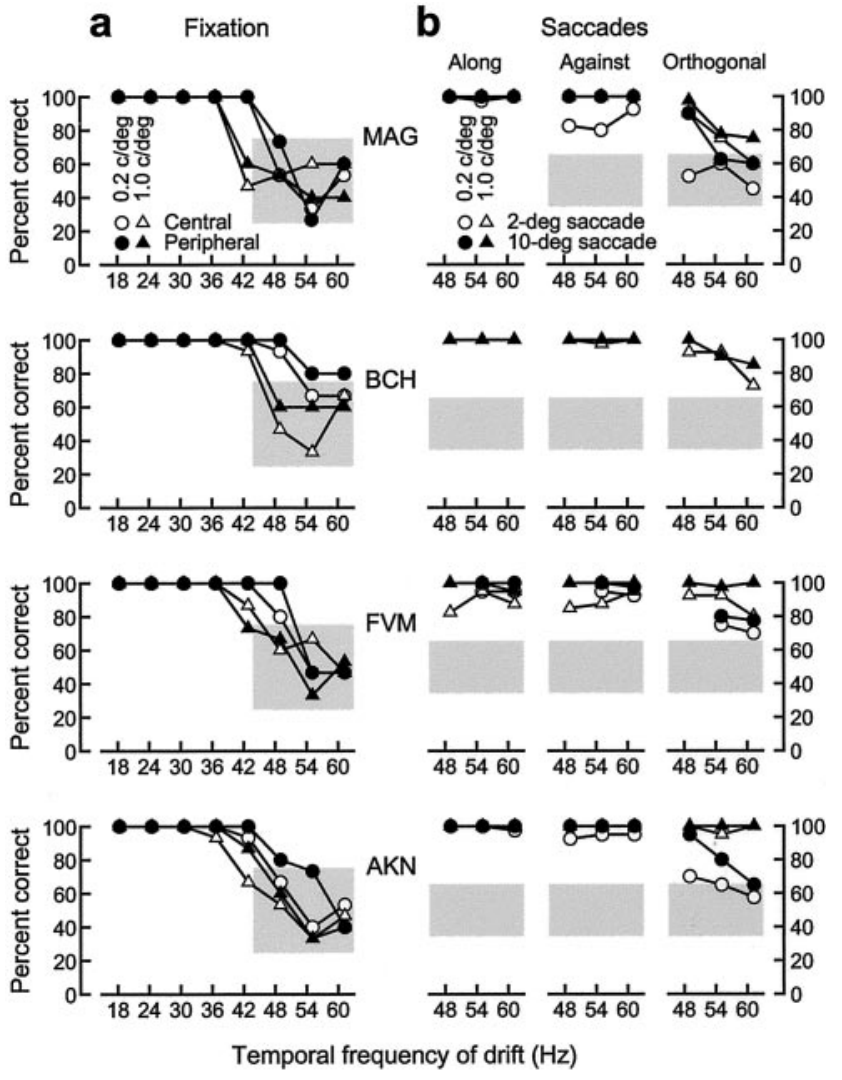

Figure 1. Visibility of gratings as a function of their temporal frequency of drift. $a$, Results under fixation, whether central (open symbols) or peripheral (solid symbols). $b$, Results during saccades along, against, and orthogonal to the direction of motion of the grating, where open and solid symbols respectively represent data for saccade amplitudes of 2 and $10^{\circ}$. In all panels, circles represent data for 0.2 cycles $/{ }^{\circ}$ gratings, triangles represent data for $1 \mathrm{cycle} /{ }^{\circ}$ gratings, and gray shading indicates the region where percentage correct does not differ significantly $(\alpha=0.05)$ from the chance level of $50 \%$. The vertical span of this region depends on the number of trials. $b$ includes only stimuli that are invisible under fixation, as indicated by chance performance in $a$, and reveals that the stimuli are detected during saccades.

frequency, detectability should and actually does increase with increasing spatial frequency and with increasing saccade amplitude (i.e., peak velocity), because either condition increases the amount of energy that spreads into the window of visibility.

Contrary to our results, it has been reported (Deubel et al., 1987; Castet and Masson, 2000) that fast-moving stimuli are not seen with saccades against their direction of motion, but this stems from the use of a higher display frame rate at which the replicas from which energy spreads toward the window of visibility lie too far above its limits. Because some of our results thus appear to be an artifact of the time-sampled operation of CRTs, we replicated the study using an apparatus that renders continuous motion.

\section{Continuous display}

When the gratings were in continuous motion, subjects never reported seeing stimuli with saccades against their direction of motion. With saccades along, performance varied with saccade amplitude and stimulus speed (Fig. 3). Saccades of a given amplitude bring into visibility stimuli drifting at all velocities below a limit that is fairly constant despite a fivefold change in spatial frequency, strikingly suggesting a velocity limit on performance.
The window-of-visibility theory (Watson et al., 1986) predicts that performance should be determined by a temporal-frequency limit, not a velocity limit. Two gratings drifting at the same velocity and differing in spatial frequency by a factor of five have their temporal-frequency content confined within ranges that are also separated by a factor of five. Saccades of a given peak velocity reduce the peak retinal velocity of either pattern by the same amount, but their corresponding temporal-frequency ranges remain separated by a factor of five. If detection requires that the temporal-frequency range of the retinal stimulus falls at least in part within the window of visibility, the curves for 1 cycle $/^{\circ}$ gratings (Fig. 3, open symbols) should be horizontally shifted to the left of the corresponding curve for 0.2 cycle $/{ }^{\circ}$ gratings (solid symbols) by the magnitude that corresponds to a factor of five, more than half of the span of the horizontal axis. We fitted a logistic function to each data set in Figure 3 and determined the lateral shift by measuring the distance between the $50 \%$ points on the 0.2 and 1 cycle/ $/{ }^{\circ}$ curves (indicated by the horizontal segments in Fig. 3). These distances imply factors ranging from 1.03 to 1.38 , with an average of 1.17 which is significantly different from 5 (one-sample $t$ test; $t_{11}=-123.37 ; p<0.00005$ ). Thus, our data do not support the prediction of the window-of-visibility theory.

Furthermore, the fact that gratings drifting at $\sim 125 \%$ sec become visible even with fast, $20^{\circ}$ saccades $(\sim 400 \% \mathrm{sec})$ seems to suggest that the process involved in their detection is not simply related to retinal velocity at the peak of the saccade (as suggested by Castet and Masson, 2000): peak retinal velocity is approximately $-275 \%$ sec in this condition, corresponding to peak temporal frequencies of $\sim 275 \mathrm{~Hz}$ (for the 1 cycle $/{ }^{\circ}$ grating) and $\sim 55$ $\mathrm{Hz}$ (for the 0.2 cycle $^{\circ}$ grating), both of which are beyond the limits of the window of visibility. All subjects reported that these gratings had the most clear appearance of a high-contrast flash of a static pattern, something that is at odds with the fact that the patterns were never even remotely close to static on the retina for any significant amount of time over the course of the saccade (Fig. 4). We will further comment on the implications of these results in our Discussion.

In a separate session we transformed the continuous display into a sampled one by replacing the DC light source with stroboscopic illumination at $333 \mathrm{~Hz}$. Subjects reported seeing the grating with every saccade, whether along or against its direction of motion. Thus, on a continuous display, fast-moving gratings are detected only when the eyes move along their direction of motion and provided their velocity does not exceed a limit that is minimally dependent on their spatial frequency. All subjects reported that the pattern did not appear to be moving, but they guessed that seeing it only during saccades in one direction would imply that it was moving in that direction. Making use of this affordable conclusion, the subjects could have reported the direction of motion of the pattern despite being unable to see motion.

\section{Intrasaccadic motion perception}

The panels on the right of Figure 2 show that the retinal stimulus during saccades contains directional information for the imputation of motion (García-Pérez and Peli, 1999). If intrasaccadic perception had access to this information, subjects should perceive motion during saccades. However, our subjects reported informally that the stimuli did not appear to move. This contrasts with the results of Castet and Masson (2000), whose subjects reported seeing motion during saccades. A possible explanation for the discrepancy is that our subjects were not paying attention to a feature that they had not been asked to identify. Another 

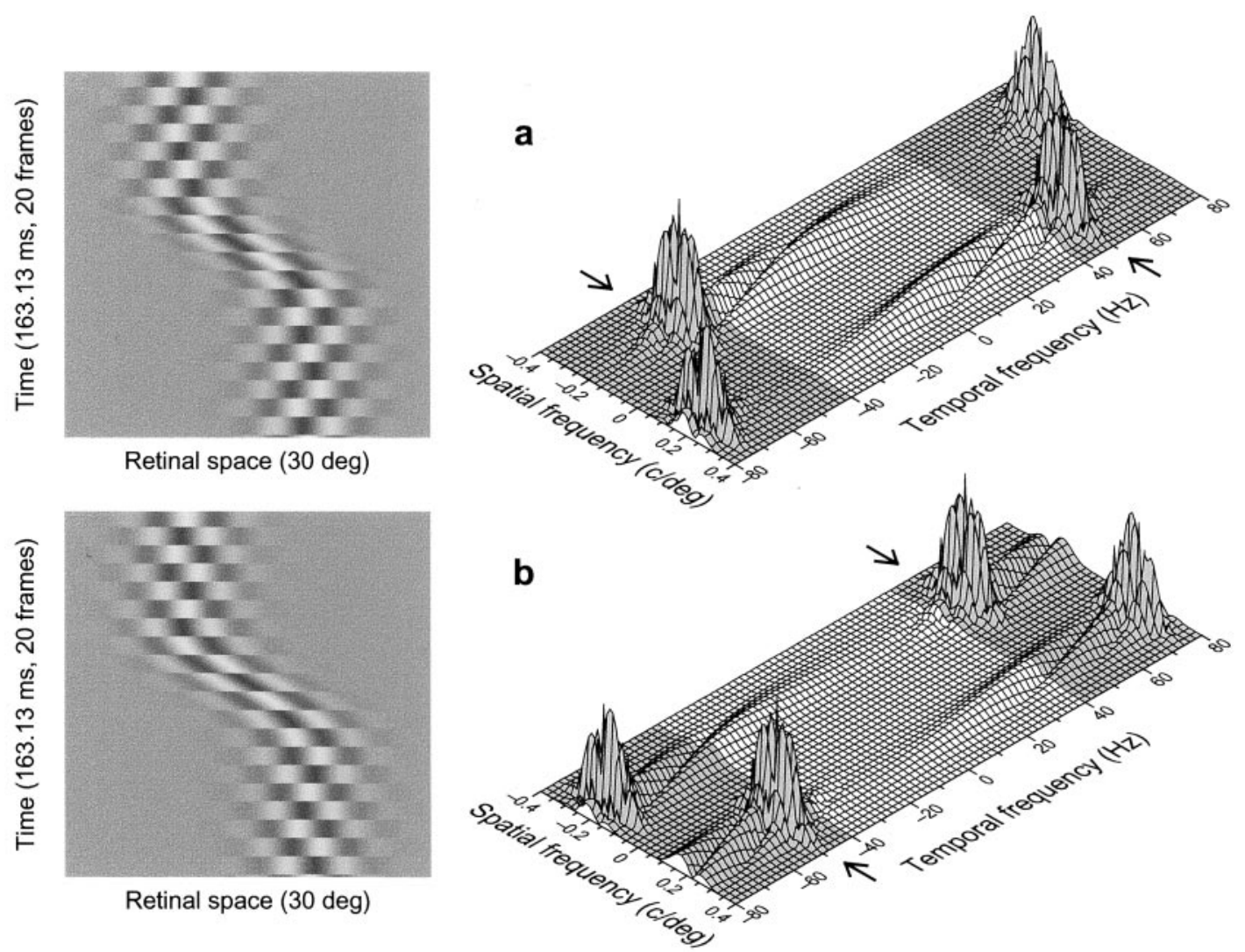

Figure 2. The retinal stimulus in the temporal vicinity of a saccade. The 0.2 cycle $/{ }^{\circ}$ grating drifts at $49.04 \mathrm{~Hz}$ (rightward motion) in $a$ and at $-49.04 \mathrm{~Hz}$ (leftward motion) in $b$. In both cases, the rightward saccade has an amplitude of $10^{\circ}$, so it is along the direction of motion of the grating in $a$ and against it in $b$. The space-time plots on the left show the retinal stimulus (the fovea is at the center of the $30^{\circ}$ horizontal span) over a brief temporal interval containing the saccade (time increases upwards), and the trajectory of the saccade is given by Equation A5 in the Appendix. The meshes on the right - with the grayed parts approximating the region beyond the window of visibility — display the corresponding amplitude spectra. Arrows indicate the blobs that correspond to the nominal stimulus; the remaining blobs are replicas produced by the time-sampled display. Saccades introduce energy into the window of visibility in both cases, regardless of the direction of the saccade with respect to the direction of motion of the grating.

possibility arises from the remark of Castet and Masson (2000) that their stimuli were only visible with saccades along their direction of motion, not with saccades against it. This is a result of their using a $160 \mathrm{~Hz}$ frame rate and a $10 \%$ contrast. Castet and Masson (2000) thus placed their subjects in a situation similar to that in our continuous display, where stimuli are detected only with saccades along their direction of motion, thus allowing motion to be guessed without seeing it. Our next experiment removes this potential contamination by using a display rate at which the stimulus is seen with saccades along and against its direction of motion (Fig. 1b) and by using a forced-choice direction-discrimination test in which subjects can only perform above chance if they actually see motion.

Intrasaccadic motion perception occurred only with $2^{\circ}$ saccades over 0.2 cycle $/{ }^{\circ}$ gratings (Fig. $5 a$ ). We thus have reasons to believe that the results of Castet and Masson (2000) indicating intrasaccadic perception of motion across a broad range of conditions are a consequence of the inappropriate mixture of display frame rate and experimental procedure. Yet, their major conclusion that motion perception may occur during saccades remains true in the light of our results. We are, however, less keen on ascribing this event to the workings of the magnocellular pathway: our subjects did not see the 0.2 cycle $/{ }^{\circ}$ grating in motion when they executed $10^{\circ}$ saccades (Fig. 5a, right column), despite the fact that this grating is processed by the magnocellular system.

Our data also suggest that intrasaccadic perception of the gratings is not mediated by a compensation of motion at the retina, because none of our subjects ever perceived reversed motion. (In a forced-choice task, reversed motion perception manifests as below-chance performance, which our data do not show) (Fig. 5a). Castet and Masson (2000) argued that saccadic overcompensation of velocity would result in the perception of reversed motion, on the assumption that perceived velocity equals peak retinal velocity, i.e., the signed difference between grating velocity and peak saccadic velocity. Also according to this assumption, the grating is seen as a static flash when the peak velocity of the saccade is close to the velocity of the grating. Castet and Masson (2000) provided empirical evidence supporting their hypothesis, but it was again based on subjective reports in yes-no tasks. Our forced-choice results provide countering evidence: the velocity of our forward-drifting gratings varied from $\sim 49 \%$ sec (for 1 cycle $/{ }^{\circ}$ at $49.04 \mathrm{~Hz}$ ) to $\sim 276 \%$ sec (for 0.2 cycles ${ }^{\circ}$ at $55.17 \mathrm{~Hz}$ ), and saccade velocities varied between $\sim 100$ and $\sim 300 \% \mathrm{sec}$, resulting in peak retinal velocities ranging from high and negative (approximately $-250 \%$ sec; backward retinal motion) to high and positive ( $\sim 175 \% \mathrm{sec}$; forward retinal motion). Our subjects perceived veridical motion in only two of these conditions, they never perceived reversed motion and most often they perceived what they informally reported as static flashes, despite the fact that peak retinal velocity for these sampled stimuli was never close to null over any significant amount of time during the saccade. 

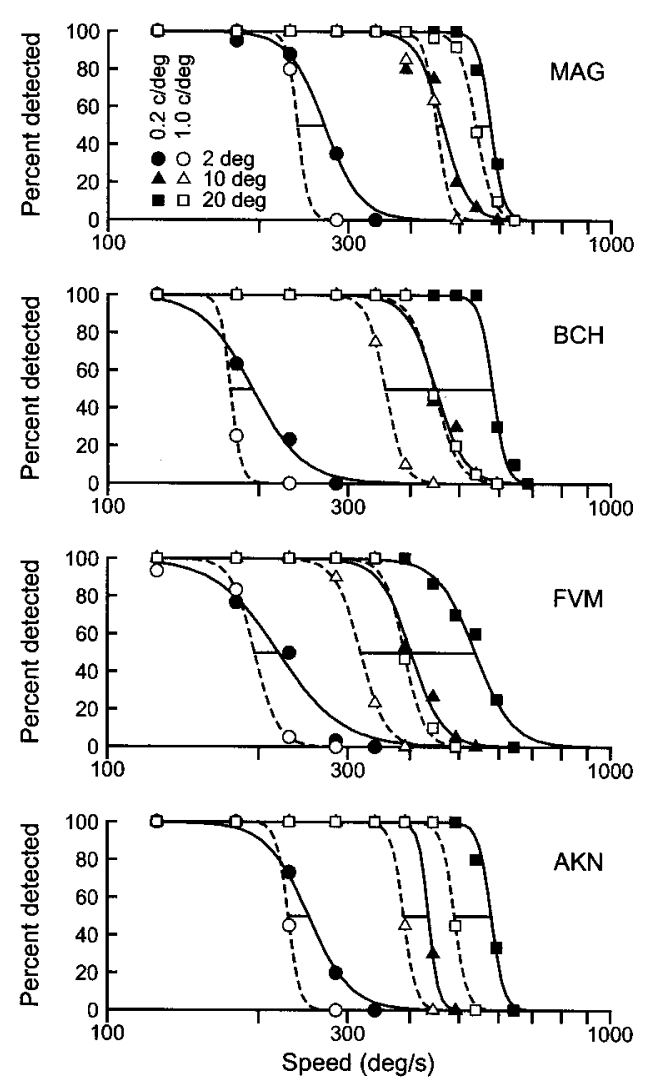

Figure 3. Visibility of gratings in continuous motion, as a function of their speed. Circles, triangles, and squares respectively represent data for 2 , 10 , and $20^{\circ}$ saccades; solid and open symbols respectively represent data for 0.2 and $1 \mathrm{cycle}^{\circ}$ gratings. All data correspond to saccades in the same direction as the stimulus motion; saccades in the opposite direction did not elicit visibility. Solid curves (for 0.2 cycle $/{ }^{\circ}$ gratings) and dashed curves (for $1 \mathrm{cycle} /{ }^{\circ}$ gratings) are the best-fitting logistic functions to each data set. The locations of the curves for 0.2 cycle $/{ }^{\circ}$ gratings and the corresponding 1 cycle $/{ }^{\circ}$ gratings, as indicated by the distance between their $50 \%$ points (horizontal segments), are not laterally shifted by the distance that corresponds to a factor of five, indicating that performance does not have a temporal-frequency limit.

\section{Direction-related differences in perceived contrast}

Our theoretical analysis of intrasaccadic visual processing (see Discussion) predicts that perceived contrast should be higher when the saccade is along than when it is against the direction of motion of the stimulus. The present experiment tests this prediction and explores whether direction-related differences in perceived contrast are limited to stimuli seen in motion during saccades or extends also to stimuli with which intrasaccadic motion is not perceived.

Stimuli drifting in the direction of the saccade were indeed generally perceived as higher in contrast (Fig. 5b). A comparison with our direction-discrimination results (Fig. $5 a$ ) reveals that this occurs for all stimuli that were seen in motion during saccades and also for stimuli not seen in motion. Differences in the perceived contrast of gratings drifting in the direction of the saccade and gratings drifting in the opposite direction reveal that intrasaccadic perception has finer grain than would be considered necessary from the theoretical standpoint that intrasaccadic visual processing is disruptive and ought to be reduced or suppressed. The next experiment further assesses the granularity of intrasaccadic contrast perception.

\section{Contrast-matching of stimuli drifting in opposite directions}

If intrasaccadic contrast perception had the broad range that it has with static eyes, subjects would be able to perform contrastmatching tasks during saccades. We checked this out by measuring the contrast that the grating moving in the direction of the saccade must have to be perceived as having the same contrast as a similar grating moving in the opposite direction. This experiment also provides quantitative data on differences in the perceived contrast of stimuli drifting in opposite directions.

The results (Fig. 6) reveal that intrasaccadic contrast perception can be well described by psychometric functions similar to those describing contrast comparisons in static viewing: the probability of perceiving one grating as having higher contrast than the other increases with distance from the point of subjective equality given by the $50 \%$ point on the fitted curve. This point (vertical lines) differs from the point of objective equality (arrows), and the difference is larger at the lower temporal frequency (solid lines, circles, and arrow).

Note that only small differences exist between retinal stimuli in the "along" and "against" conditions (Fig. 2): the most salient characteristic (which both situations share) is that the saccade introduces the same continuous motion during each of the otherwise static display frames (since both gratings were presented simultaneously and, then, both were affected by exactly the same saccade-induced motion). The only minor difference that remains is the spatial offset that each new display frame introduces. This minor difference must cause the observed differences in perceived contrast. The classical explanation for the invisibility of highfrequency flicker and fast-moving gratings under fixation is that temporal contrast is blurred away by a temporal integration process (Levinson, 1968). If this process also operates during saccades, the perceived contrast of stimuli drifting in opposite directions must reflect the different outcomes of this process. We present theoretical results supporting this hypothesis in the last section of our forthcoming discussion.

\section{DISCUSSION}

\section{The status of saccadic suppression}

Intrasaccadic perception argues against saccadic suppression as an active process affecting suprathreshold vision. Research on saccadic suppression has focused on threshold perception, and it has also generally failed to design conditions that unequivocally indicate whether lower sensitivity during saccades is indeed the result of degraded processing. A comparison between thresholds under fixation and during saccades is biased, because the stimulus is hardly the same in both cases: in fixation trials it falls onto a single retinal location for its entire duration, whereas in saccade trials it is broadly spread across a larger retinal area. Each of the retinal areas involved during saccade trials receives a weaker stimulation for a shorter time, and multiple factors will produce a threshold elevation in these conditions: retinal inhomogeneity, Bloch's law (Gorea and Tyler, 1986), exposure duration (Tulunay-Keesey and Jones, 1976), temporal integration (Burr, 1981), and probability summation (Watson, 1979; Robson and Graham, 1981).

A careful study has recently been published that overcomes these difficulties by comparing the threshold effects of real and simulated saccades (Diamond et al., 2000). The authors claimed that suppression occurs only during real saccades, although one of the experiments remarkably showed the same suppression during 
2-deg saccade

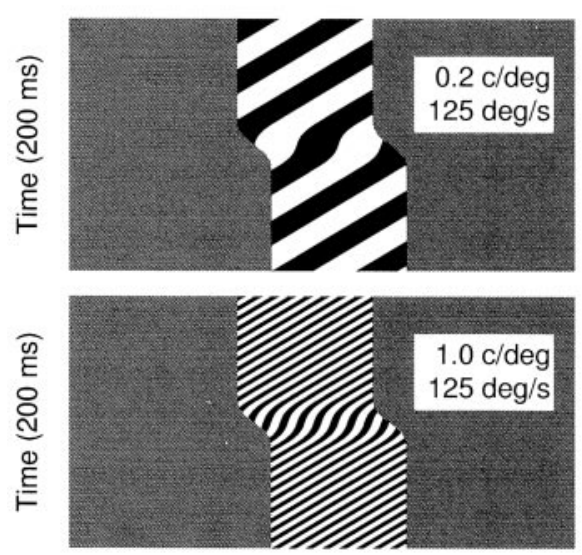

Retinal space (30 deg) 10-deg saccade
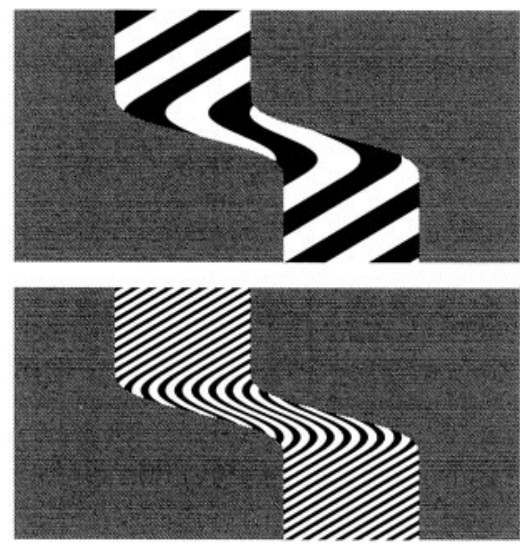

Retinal space (30 deg) 20-deg saccade
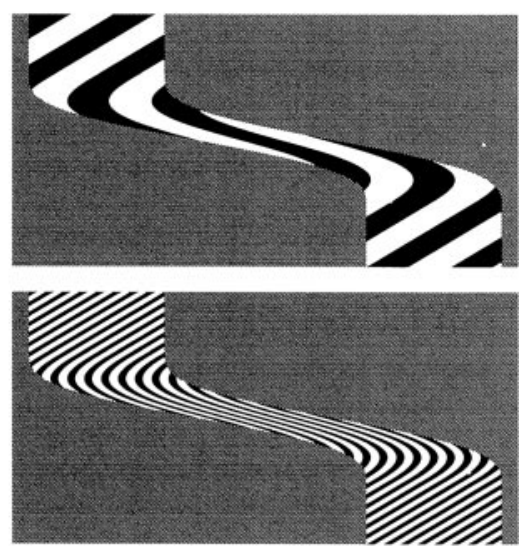

Retinal space (30 deg)

Figure 4. Retinal projection of square-wave gratings drifting in continuous motion. Saccade trajectories are given by Equation A5 in the Appendix. Top shows 0.2 cycle $/{ }^{\circ}$ grating drifting at $125 \%$ sec; bottom, 1 cycle $/{ }^{\circ}$ grating drifting at $125 \%$ sec. Left shows $2^{\circ}$ saccade (duration, $\delta=0.044$ sec; peak velocity, $99.43 \% \mathrm{sec})$; center, $10^{\circ}$ saccade $(\delta=0.073 \mathrm{sec}$; peak velocity, $299.66 \% \mathrm{sec})$; right, $20^{\circ}$ saccade $(\delta=0.109 \mathrm{sec}$; peak velocity, $401.38 \%$ sec $)$. Retinal motion is continuous, and gratings are never static on the retina over any significant amount of time.

real and simulated saccades (Diamond et al., 2000, their Fig. 5). This contradictory evidence demands further research, a research that should also solve a minor methodological problem described next.

In the experiments of Diamond et al. (2000), the task was one of luminance-change detection, because the study used a Gabor patch whose space constant was $<1 / 5$ the grating period: presentation of the patch then resulted mainly in a luminance change. Also, $0.5^{\circ}$ fixation spots were used that provided a contaminating local contrast clue, whose effect was maximal because spots were located along the line of maximal luminance. Finally, in trials with real saccades the subjects moved their eyes from one of these spots to the other, whereas in trials with simulated saccades a mirror moved the entire display in the opposite direction, away from fixation spots (Diamond et al., 2000, their Fig. 1). Then, real and simulated saccades differed in that a fixation spot was foveally available after real saccades but not after simulated saccades.

These details may explain the minor difference that was found between real and simulated saccades when the task was detection against a structured background, namely, a "slower recovery of sensitivity in the no-saccade condition" (Diamond et al., 2000, p. 3454). This outcome seems to reflect that, for stimuli displayed after simulated saccades, subjects could not use the foveal contrast clue that facilitated detection after real saccades. It is uncertain why suppression was specific to real saccades in other cases, but the lack of specificity under some conditions indicates that active saccadic suppression is not always operative. Being diurnal mammals, our visual system has evolved to deal with high contrast and structured backgrounds. The demonstration of the lack of saccadic suppression with structured backgrounds of Diamond et al. (2000) and our demonstration of intrasaccadic perception of high-contrast stimuli support the idea that saccadic suppression does not play any role under the conditions in which our visual system has evolved.

\section{Motion perception during saccades}

Castet and Masson (2000) hypothesized that intrasaccadic motion perception occurs for stimuli that are optimal for the magnocellular system (low spatial and high temporal frequencies) if retinal temporal frequency at the peak of the saccade is within the optimal range for motion detection. If $v_{p}$ is this peak velocity (in degrees per second) and $\omega_{g}$ is the temporal frequency (in Hertz) of a grating with a spatial frequency of $\rho_{g}$ cycles $^{\circ}{ }^{\circ}$, the retinal temporal frequency at the peak of the saccade is $\omega_{r}=\omega_{g}-v_{p} \rho_{g}$. Accordingly, $2^{\circ}$ saccades yielding $v_{p} \approx 100 \% / \mathrm{sec}$ should elicit veridical motion perception when $\rho_{g}=0.2$ cycles $/{ }^{\circ}$ and $\omega_{g}=49.04$ or $55.17 \mathrm{~Hz}$ (so that $\omega_{r} \approx 29.04$ or $35.17 \mathrm{~Hz}$ ), and our empirical data indicate that this is the case (Fig. $5 a$ ). However, $2^{\circ}$ saccades should also elicit veridical motion perception when $\rho_{g}=0.5$ cycles $/{ }^{\circ}$ and $\omega_{g}=55.17 \mathrm{~Hz}$ (so that $\omega_{r} \approx 5.17 \mathrm{~Hz}$ ), and our data indicate this is not the case (Fig. $5 a$ ). Similarly, $10^{\circ}$ saccades yielding $v_{p} \approx 300 \% \mathrm{sec}$ should elicit reversed motion perception when $\rho_{g}=0.2$ cycles $^{\circ}$ and $\omega_{g}=49.04$ or $55.17 \mathrm{~Hz}$ (so that $\omega_{r} \approx$ -10.96 or $-4.83 \mathrm{~Hz}$ ), but our empirical data also disconfirm this prediction (Fig. $5 a$ ).

Our results indicate that intrasaccadic motion perception does not depend on isolated events at around the time that the saccade reaches peak velocity.

\section{Visual processes mediating intrasaccadic perception}

Deubel et al. (1987) suggested that intrasaccadic detection might be either a result of reduced retinal velocity or a result of the temporal transient that occurs when the image "disappears" from one retinal location and "reappears" on another one. The first possibility was also considered by Castet and Masson (2000), but our data disprove it: our subjects detected gratings whose retinal velocity at the peak of the saccade is not within the window of visibility. The second possibility lines up with a recent hypothesis stating that the unexpected flash of a stimulus resets motion integration (Eagleman and Sejnowski, 2000), a hypothesis that seems to account for the flash-lag effect (Nijhawan, 1994). This hypothesis seems also disproved by our results, because orthogonal saccades yield poorer performance than saccades along or against the direction of motion of the stimulus (Fig. 1b). A third possibility, namely, that temporal integration processes taking place locally in space are also operative during saccades, makes predictions that are consistent with our results.

Consider the temporal impulse response (TIR) of the visual 


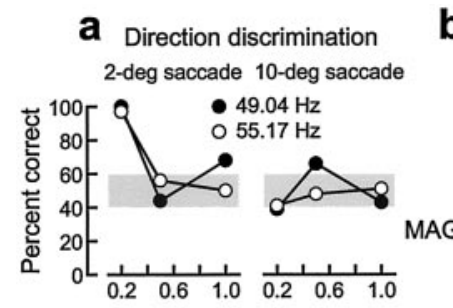

b Contrast discrimination 2-deg saccade 10-deg saccade

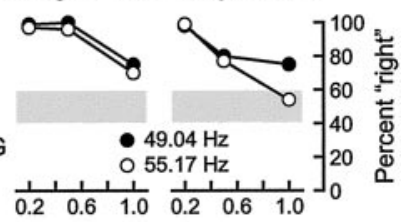

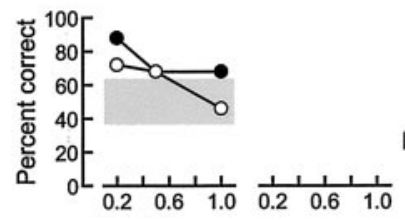

$\mathrm{BCH}$
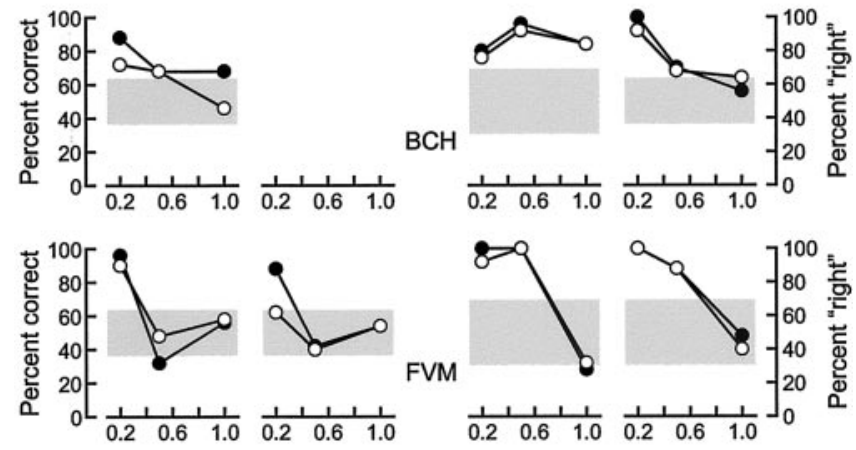

FVM
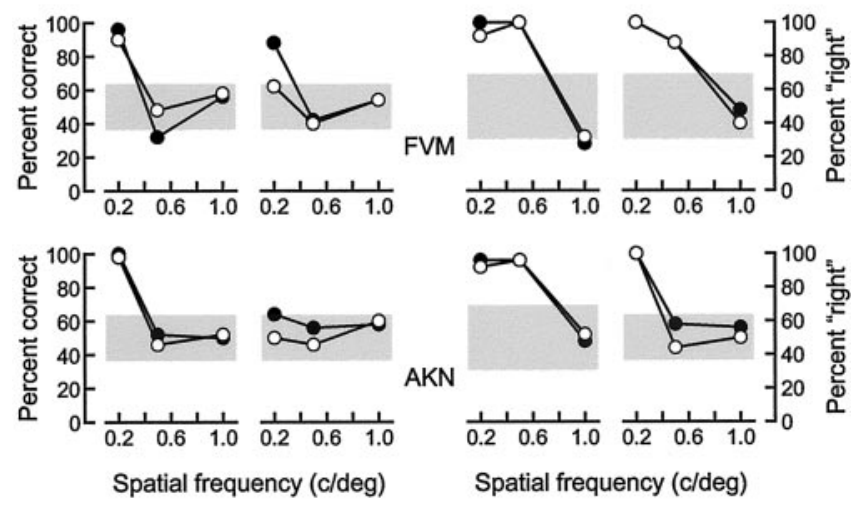

Figure 5. Performance in the direction-discrimination and contrastdiscrimination tests. Solid and open circles respectively represent data for 49.04 and $55.17 \mathrm{~Hz}$ drift. Gray shading indicates the region where percentage correct (or percentage "right") does not differ significantly $(\alpha=$ 0.05 ) from the chance level of $50 \%$. a, Intrasaccadic motion perception only occurs with $2^{\circ}$ saccades over 0.2 cycle $/{ }^{\circ}$ gratings. Subject $\mathrm{BCH}$ found the direction-discrimination task with $10^{\circ}$ saccades so difficult that halfway through the session she started executing smaller saccades that made the task easier; she could not prevent this from happening, and her data were discarded, but she was essentially performing at chance level. $b$, Although gratings drifting along and against the direction of the saccade had the same physical contrast, the grating drifting to the right (i.e., in the same direction as the saccade) was perceived to have a higher contrast in a number of conditions including that sustaining intrasaccadic motion perception.

system to be the difference between $n_{1}$ stage and $n_{2}$ stage filters (Bergen and Wilson, 1985; Watson and Ahumada, 1985),

$g(t)$

$$
=\left\{\begin{array}{cc}
\frac{a\left(t / \tau_{1}\right)^{n_{1}-1} \exp \left(-t / \tau_{1}\right)}{\tau_{1}\left(n_{1}-1\right) !}-\frac{b\left(t / \tau_{2}\right)^{n_{2}-1} \exp \left(-t / \tau_{2}\right)}{\tau_{2}\left(n_{2}-1\right) !} & \text { if } t>0 \\
0 & \text { otherwise. }
\end{array}\right.
$$

To illustrate the effects of temporal integration over the period containing a saccade, we will use $n_{1}=9, n_{2}=10, a=1, b=0.9$, $\tau_{1}=0.004 \mathrm{sec}$, and $\tau_{2}=0.0053 \mathrm{sec}$ (Watson and Ahumada, 1985) (Fig. 7).

Assuming that temporal integration takes place whether or not the eyes are static, the outcome of this process is given by temporal convolution of the TIR and the retinal stimulus, which may include shifts caused by saccades. Parameter $b$ in Equation 1 mostly determines whether the resulting TIR has a bandpass or a lowpass characteristic which, in turn, determines whether the

\section{a $\quad 0.5 \mathrm{c} / \mathrm{deg}, 2-\mathrm{deg}$ saccades} $\mathbf{b}_{0.2 \mathrm{c} / \mathrm{deg}, 10-\mathrm{deg} \text { saccades }}$
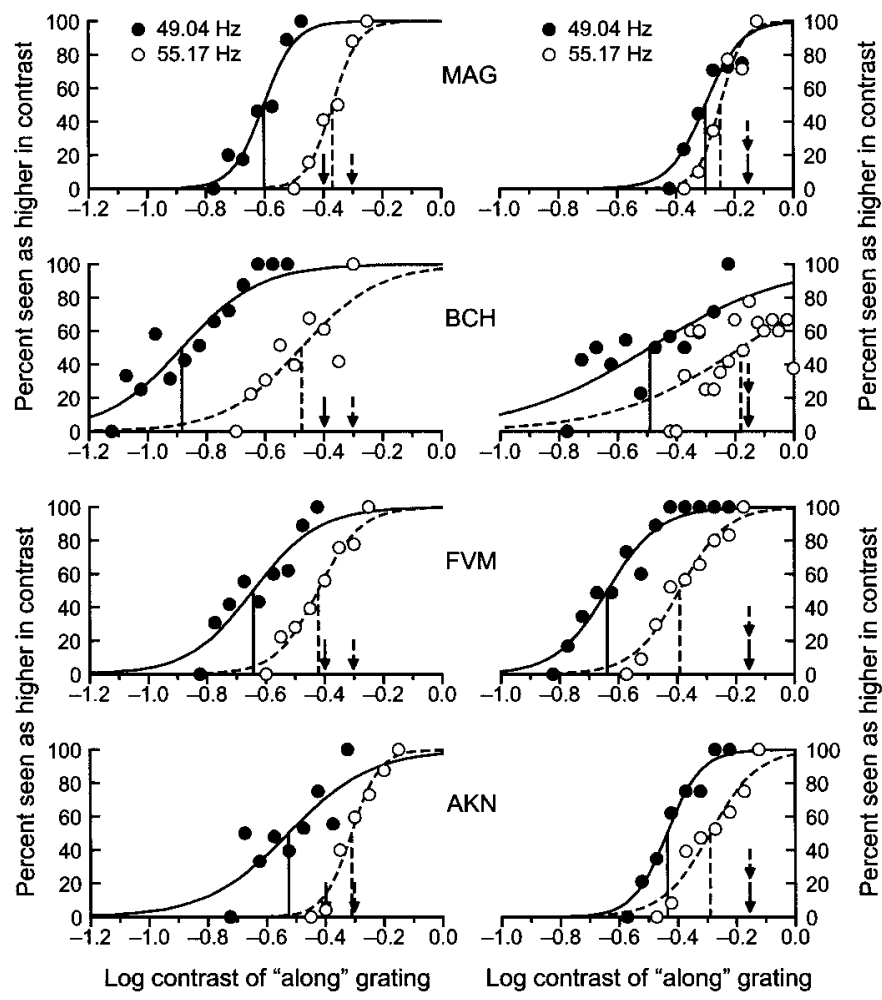

Figure 6. Performance in the contrast-matching test. Data indicate the percentage of times that a grating drifting in the direction of a saccade was perceived as having higher contrast than a grating drifting in the opposite direction, as a function of the contrast of the former. The contrast of the grating drifting in the opposite direction was fixed at the value indicated by the arrows. Solid and open circles respectively represent data for drift at 49.04 and $55.17 \mathrm{~Hz}$; solid and dashed curves represent maximum likelihood fits to the corresponding data, and their $50 \%$ point (vertical lines) is an estimate of the contrast at which the "along" grating is perceived to have the same contrast as the "against" grating. This matching contrast is lower than the actual contrast of the "against" grating, and the difference is larger at the lower temporal frequency (solid symbols, solid lines, and solid arrow). $a$, Results for 0.5 cycle $/{ }^{\circ}$ gratings and $2^{\circ}$ saccades. $b$, Results for 0.2 cycle ${ }^{\circ}$ gratings and $10^{\circ}$ saccades.

output will include what is referred to as "off" responses in the neurophysiological literature (García-Pérez, 1999). "Off” responses are the temporal (causal) analog of lateral inhibition in space, and they explain why gratings briefly flashed in sequence summate better in counterphase (Watson and Nachmias, 1977). The following results do not depend on $b$.

Temporal integration blurs away contrast before and after saccades-consistent with the invisibility of stimuli under fixation - and only the retinal stimulus over a brief period around the saccade survives the integration-also consistent with the visibility of stimuli during saccades. For stimuli displayed on a CRT, non-null output occurs for saccades both along (Fig. 8, left) and against (Fig. 8, right) its direction of motion, but there are quantitative differences between the two conditions. The maximal instantaneous spatial contrast is generally higher for saccades in the direction of stimulus motion, consistent with our contrastmatching results (Fig. 6): the difference is larger at the lower temporal frequency.

Also consistent with our results in Figure 3, the output for stimuli in continuous motion (data not shown) is null for saccades 


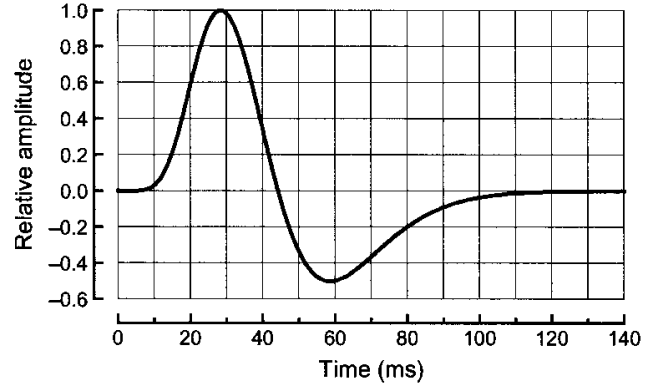

Figure 7. Shape of the TIR in Equation 1 with parameters from Watson and Ahumada (1985). This TIR consists of an initial delay of $\sim 10 \mathrm{msec}$ followed by a positive lobe that spans $\sim 35 \mathrm{msec}$, determining the time over which temporal oscillations in the stimulus will be blurred away. The subsequent negative lobe spans $\sim 65 \mathrm{msec}$ and will produce an "off" response. If the temporal input were a single, above-threshold flash of infinitesimal duration, the temporal output would have the shape described by this function: a slightly delayed flash-like appearance followed by a short and weaker contrast-reversed version (the "off" response).

against the direction of stimulus motion, whereas saccades along that direction produce similar output as with the sampled display.

Temporal integration also explains the puzzling velocity limit on performance (Fig. 3). The stimulus is blurred away before and after the saccade because its fast temporal variations are filtered out at all spatial locations. Yet, during saccades the retinal stimulus has "elbows" that extend the time over which any retinal location receives stimulation of the same polarity (Fig. 4), and this perturbation passes through the temporal filter. The duration of this transient is not well described in temporal-frequency terms and depends mostly on grating velocity (hence the velocity limit) and minimally on spatial frequency: all else equal, the duration of the perturbation increases slightly as spatial frequency decreases, consistent with our result that the velocity limit is slightly higher for 0.2 than for $1 \mathrm{cycle}^{\circ}$ gratings (Fig. 3). Variations in the duration of this perturbation are too small to be noticed (consistent with the flash-like appearance reported by our subjects), and the two occasions of its occurrence (at the beginning and at the end of the saccade) are merged into a single event by the spread of the TIR: the perturbation at the output is a single event with a fairly constant duration of $50-100 \mathrm{msec}$.

Our results thus suggest that visual processing operates during saccades in much the same way as under fixation, and failure to notice intrasaccadic perception is likely a result of visual masking caused by presaccadic and postsaccadic perception (Campbell and Wurtz, 1978; Corfield et al., 1978).

\section{APPENDIX: THE RETINAL STIMULUS DURING SACCADES}

During a saccade, the distal stimulus sweeps the retina according to the path of the eye movement. Let $f$ be the distal stimulus, and consider for simplicity that it is a Gabor function whose Gaussian aperture has a circular spatial spread of $\sigma^{\circ}$ and whose carrier has a spatial frequency of $\rho_{0}$ cycles $/{ }^{\circ}$ and a velocity of $v_{0} \%$ sec and that is further windowed with a temporal contrast envelope that spans $t_{0}$ sec and whose onset and offset are linearly ramped for $\tau_{0} \sec \left(\tau_{0}\right.$ $\leq t_{0} / 2$ ). Assuming foveal presentation and foveal spatial coordinates, and setting the arbitrary origin of time at stimulus onset, the nominal stimulus is

$f(x, y, t)$

$$
=L_{0}\left\{1+m(t) \exp \left[-\frac{x^{2}+y^{2}}{2 \sigma^{2}}\right] \cos \left[2 \pi \rho_{0}\left(x-v_{0} t\right)\right]\right\},
$$

where $L_{0}$ is mean luminance and

$$
m(t)=\left\{\begin{array}{cc}
m_{\max } \frac{t}{\tau_{0}} & \text { if } 0 \leq t<\tau_{0} \\
m_{\max } & \text { if } \tau_{0} \leq t<t_{0}-\tau_{0} \\
m_{\max } \frac{t_{0}-t}{\tau_{0}} & \text { if } t_{0}-\tau_{0} \leq t \leq t_{0} \\
0 & \text { otherwise }
\end{array}\right.
$$

is the temporal contrast envelope, with maximal contrast $m_{\max }$.

When displayed on a CRT at a frame rate of $\omega_{0} \mathrm{~Hz}$, the actual stimulus $f_{a}$ consists of a discrete sequence of $n$ static frames, where $n=\left[t_{0} \omega_{0}\right]$ (i.e., the least integer value greater than or equal to $\left.t_{0} \omega_{0}\right)$. Apparent motion occurs because the carrier shifts in space across frames, although it remains static over the entire duration of a frame. Disregarding the mean luminance pedestal, the actual stimulus is then:

$$
f_{a}(x, y, t)=L_{0} \sum_{i=1}^{n} m_{i} \Pi_{i}(t) \exp \left[-\frac{x^{2}+y^{2}}{2 \sigma^{2}}\right] \cos \left[2 \pi \rho_{0}\left(x-x_{i}\right)\right],
$$
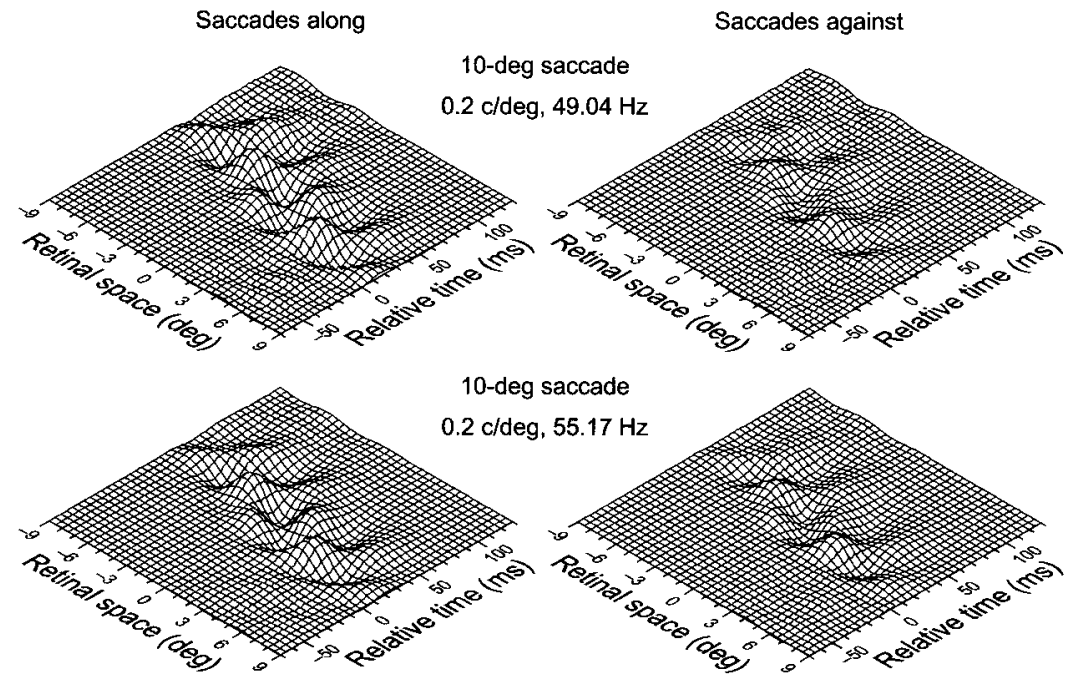

10-deg saccade

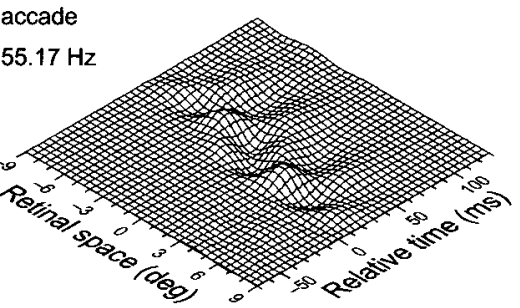

Figure 8. Output of a temporal integration process operating at each retinal location. The input is the retinal stimulus resulting from saccades of given amplitudes over gratings of given spatial and temporal frequencies, as rendered on the time-sampled display used in our experiments (Fig. 2). The origin of the temporal axis is at the midpoint of the saccade, when saccadic velocity peaks. The delay of the temporal perturbation produced by the saccade with respect to the saccade itself is determined by the shape of the causal TIR (Fig. 7). The top panels pertain to the stimuli in Figure 2. The maximal instantaneous spatial contrast at the output is generally larger in the "along" condition (left column) than in the corresponding "against" condition (right column), and the difference is larger at the lower temporal frequency (top), in agreement with our intrasaccadic contrast-matching results (Fig. 6). 
where $m_{i}=m\left((i-1) / \omega_{0}\right)$ is the contrast of the Gabor function displayed on the $i$ th frame, $x_{i}=v_{0}(i-1) / \omega_{0}$ is the spatial shift of the carrier at the $i$ th frame, and

$$
\Pi_{i}(t)=\left\{\begin{array}{cc}
1 & \text { if } \frac{i-1}{\omega_{0}} \leq t \leq \frac{i}{\omega_{0}} \\
0 & \text { otherwise }
\end{array}\right.
$$

is the temporal window that describes the duration of the $i$ th frame. We will assume that the stimuli are displayed in conditions that minimize artifacts caused by interactions along raster lines, by phosphor decay over the duration of a frame, and by phosphor persistence across frames (García-Pérez and Peli, 2001).

When a saccade occurs whose midpoint is at time $t_{s}\left(t_{s}<t_{0}\right)$, the stimulus changes retinal position continuously over time as a result of the saccadic trajectory, and the retinal stimulus $f_{r}$ is given by $f_{r}(x, y, t)=f_{a}\left(x-x^{\prime}\left(t-t_{\mathrm{s}}\right), y-y^{\prime}\left(t-t_{\mathrm{s}}\right), t\right)$, where $x^{\prime}$ and $y^{\prime}$ are parametric functions, respectively, describing the trajectory of the saccade in the horizontal and vertical directions. Here we will assume $y^{\prime}(t)=0$ and will thus only consider horizontal saccades whose trajectory is given by

$$
\begin{aligned}
& x^{\prime}(t) \\
& =\left\{\begin{array}{ccc}
A & \text { if } \quad t \leq-\frac{\delta}{2} \\
\frac{B+A}{2}+(B-A)\left(\frac{35 t}{16 \delta}-\frac{35 t^{3}}{4 \delta^{3}}+\frac{21 t^{5}}{\delta^{5}}-\frac{20 t^{7}}{\delta^{7}}\right) & \text { if } & -\frac{\delta}{2}<t \leq \frac{\delta}{2}, \\
B & \text { if } & t>\frac{\delta}{2}
\end{array}\right.
\end{aligned}
$$

where $B-A$ (in degrees) is the amplitude of a saccade that changes the retinal location of the stimulus from $A$ to $B(A<B$ for rightward saccades; $A>B$ for leftward saccades) and $\delta$ (in seconds) is its duration (Fig. 9). This sigmoidal trajectory corresponds to a minimum-snap model (Harwood et al., 1999), and it is easy to show that the velocity of such saccade is given by:

$$
v_{s}(t)=\frac{d x^{\prime}}{d t}=\left\{\begin{array}{cc}
\frac{35(B-A)}{16 \delta}\left(1-\frac{4 t^{2}}{\delta^{2}}\right)^{3} & \text { if }-\frac{\delta}{2} \leq t \leq \frac{\delta}{2}, \\
0 & \text { otherwise }
\end{array}\right.
$$

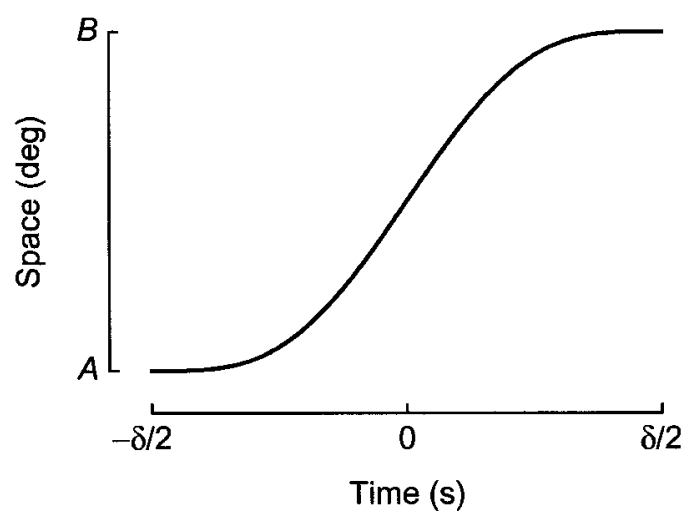

Figure 9. Sigmoidal trajectory of a saccade as given by Equation A5. The arbitrary origin of time is at the midpoint of the saccade, when velocity is at its peak. yielding a peak velocity $v_{p}=35(B-A) / 16 \delta \%$ sec at the midpoint of the saccade (i.e., peak velocity varies inversely with duration and directly with amplitude).

It is also useful to look at this retinal stimulus in the spatiotemporal-frequency domain, since it gives some insight as to the effect of saccades on the spectral content of the retinal stimulus. The functional form of $x^{\prime}$ does not permit obtaining the Fourier transform of $f_{r}$, in closed form, but an analytical approximation can be obtained to the desired precision by noting that $x^{\prime}$ can be approximated through a stepwise linear function with constant-velocity segments of appropriate durations. Thus, consider that the duration of a display frame is partitioned into adjacent epochs each lasting $\delta_{0}=1 / k \omega_{0}$ sec, with $k$ sufficiently large so that the velocity of the eye over each epoch can be considered constant. The retinal stimulus can then be formally represented as

$$
f_{r}(x, y, t)
$$

$$
\begin{aligned}
= & L_{0} \sum_{i=1}^{n} m_{i} \Pi_{i}(t) \exp \left[-\frac{\left(x-x^{\prime}\left(t-t_{s}\right)\right)^{2}+y^{2}}{2 \sigma^{2}}\right] \\
& \times \cos \left[2 \pi \rho_{0}\left(x-x^{\prime}\left(t-t_{s}\right)-x_{i}\right)\right] \\
\approx & L_{0} \sum_{i=1}^{n} \sum_{j=1}^{k} m_{i} \hat{\Pi}_{i j}(t) \exp \left[-\frac{\left(x-x_{i j}^{\prime}-v_{i j}\left(t-t_{i j}\right)\right)^{2}+y^{2}}{2 \sigma^{2}}\right] \\
& \times \cos \left[2 \pi \rho_{0}\left(x-x_{i j}^{\prime}-v_{i j}\left(t-t_{i j}\right)-x_{i}\right)\right],
\end{aligned}
$$

where

$$
\hat{\Pi}_{i j}(t)=\left\{\begin{array}{cc}
1 & \text { if } t_{i j} \leq t \leq t_{i j}+\delta_{0} \\
0 & \text { otherwise }
\end{array}\right.
$$

with $t_{i j}=(i-1) / \omega_{0}+(j-1) \delta_{0}$, defines the $j$ th epoch of the $i$ th frame, $x_{i j}^{\prime}=x^{\prime}\left(t_{i j}-t_{s}\right)$ is the location of the stimulus at the beginning of that epoch, and

$$
v_{i j}=\frac{x^{\prime}\left(t_{i j}+\delta_{0}-t_{s}\right)-x^{\prime}\left(t_{i j}-t_{s}\right)}{\delta_{0}}
$$

is the (constant) velocity of the stimulus over that epoch. The Fourier transform $F_{r}$ of the latter expression for $f_{r}$ can easily be shown to be

$$
\begin{aligned}
& F_{r}\left(\rho_{x}, \rho_{y}, \omega\right) \\
& \approx L_{0} \sum_{i=1}^{n} \sum_{j=1}^{k} m_{i} \sigma^{2} \frac{\sin \left[\pi \delta_{0}\left(\omega+v_{i j} \rho_{x}\right)\right]}{\omega+v_{i j} \rho_{x}} \exp \left[-I \pi\left(\delta_{0}+2 t_{i j}\right)\left(\omega+v_{i j} \rho_{x}\right)\right] \\
& \quad \times\left\{\exp \left[-2 \pi^{2} \sigma^{2}\left(\left(\rho_{x}-\rho_{0}\right)^{2}+\rho_{y}^{2}\right)-I 2 \pi\left(\left(x_{i j}^{\prime}-v_{i j} t_{i j}\right) \rho_{x}+x_{i} \rho_{0}\right)\right]\right. \\
& \left.\quad+\exp \left[-2 \pi^{2} \sigma^{2}\left(\left(\rho_{x}+\rho_{0}\right)^{2}+\rho_{y}^{2}\right)-I 2 \pi\left(x_{i j}^{\prime}-v_{i j} t_{i j}\right) \rho_{x}+x_{i} \rho_{0}\right)\right]
\end{aligned}
$$

where $I^{2}=-1$.

The space-time aspect (Eq. A7) and amplitude spectrum (modulus of Eq. A10) of some stimuli during saccades are shown in Figure 2. In space-time, saccades have the effect of introducing continuous motion into the stimulus: what otherwise would be static within-frame stimulation gets swept across the retina continuously, and it is this additional motion introduced by the 
saccade itself that is responsible for the temporal-frequency spread of energy. This within-frame motion also contributes to strengthening a local temporal signal caused by the abrupt phase shifts across frames.

\section{REFERENCES}

Bergen JR, Wilson HR (1985) Prediction of flicker sensitivities from temporal three-pulse data. Vision Res 25:577-582.

Burr DC (1981) Temporal summation of moving images by the human visual system. Proc R Soc Lond B Biol Sci 211:321-339.

Burr DC, Ross J (1982) Contrast sensitivity at high velocities. Vision Res 22:479-484.

Burr DC, Morrone MC, Ross J (1994) Selective suppression of the magnocellular visual pathway during saccadic eye movements. Nature 371:511-513.

Campbell FW, Wurtz RH (1978) Saccadic omission: why we do not see a grey-out during a saccadic eye movement. Vision Res 18:1297-1303.

Castet E, Masson GS (2000) Motion perception during saccadic eye movements. Nat Neurosci 3:177-183.

Corfield R, Frosdick JP, Campbell FW (1978) Grey-out elimination: the roles of spatial waveform, frequency and phase. Vision Res 18:1305-1311.

Deubel H, Elsner T, Hauske G (1987) Saccadic eye movements and the detection of fast-moving gratings. Biol Cybern 57:37-45.

Diamond MR, Ross J, Morrone MC (2000) Extraretinal control of saccadic suppression. J Neurosci 20:3449-3455.

Eagleman DM, Sejnowski TJ (2000) Motion integration and postdiction in visual awareness. Science 287:2036-2038.

Flipse JP, van der Wildt GJ, Rodenburg M, Keemink CJ, Knol PGM (1988) Contrast sensitivity for oscillating sine wave gratings during ocular fixation and pursuit. Vision Res 28:819-826.

García-Pérez MA (1999) Direction selectivity and spatiotemporal separability in simple cortical cells. J Comput Neurosci 7:173-189.

García-Pérez MA, Peli E (1999) Imputation of direction of motion in one dimension. J Opt Soc Am A 16:1531-1540.

García-Pérez MA, Peli E (2001) Luminance artifacts of cathode-ray tube displays for vision research. Spat Vis 14:201-215.

Gorea A, Tyler CW (1986) New look at Bloch's law for contrast. J Opt Soc Am A 3:52-61.

Harwood MR, Mezey LE, Harris CM (1999) The spectral main sequence of human saccades. J Neurosci 19:9098-9106.

Kelly DH (1972) Adaptation effects on spatio-temporal sine-wave thresholds. Vision Res 12:89-101.
Kelly DH (1979) Motion and vision. II. Stabilized spatio-temporal threshold surface. J Opt Soc Am 69:1340-1349.

Kelly DH (1984) Retinal inhomogeneity. I. Spatiotemporal contrast sensitivity. J Opt Soc Am A 1:107-113.

Kelly DH (1990) Moving gratings and microsaccades. J Opt Soc Am A 7:2237-2244.

Koenderink JJ, van Doorn AJ (1979) Spatiotemporal contrast detection threshold surface is bimodal. Opt Lett 4:32-34.

Kulikowski JJ (1971) The effect of eye movements on the contrast sensitivity of spatio-temporal patterns. Vision Res 11:261-273.

Levinson JZ (1968) Flicker fusion phenomena. Science 160:21-28.

Matin E (1974) Saccadic suppression: a review and an analysis. Psychol Bull 81:899-917.

Murphy BJ (1978) Pattern thresholds for moving and stationary gratings during smooth eye movement. Vision Res 18:521-530.

Nijhawan R (1994) Motion extrapolation in catching. Nature 370:256257.

Peli E, Labianca AT, Fine EM (1998) The detection of moving features on a display: the interaction of direction of motion, orientation, and display rate. SiD Digest 29:1033-1036.

Robson JG (1966) Spatial and temporal contrast-sensitivity functions of the visual system. J Opt Soc Am 56:1141-1142.

Robson JG, Graham N (1981) Probability summation and regional variation in contrast sensitivity across the visual field. Vision Res 21:409-418.

Rottach KG, Das VE, Wohlgemuth W, Zivotofsky AZ, Leigh RJ (1998) Properties of horizontal saccades accompanied by blinks. J Neurophysiol 79:2895-2902.

Swift D, Panish S, Hippensteel B (1997) The use of VisionWorks in visual psychophysics research. Spat Vis 10:471-477.

Tulunay-Keesey U, Jones RM (1976) The effect of micromovements of the eye and exposure duration on contrast sensitivity. Vision Res 16:481-488.

van Ness FL, Koenderink JJ, Nas H, Bouman MA (1967) Spatiotemporal modulation transfer in the human eye. J Opt Soc Am 57:1082-1088.

Volkmann FC (1986) Human visual suppression. Vision Res 26:1401-1416.

Watson AB (1979) Probability summation over time. Vision Res 19:515522.

Watson AB, Ahumada Jr AJ (1985) Model of human visual-motion sensing. J Opt Soc Am A 2:322-342.

Watson AB, Nachmias J (1977) Patterns of temporal interaction in the detection of gratings. Vision Res 17:893-902.

Watson AB, Ahumada Jr AJ, Farrell JE (1986) Window of visibility: a psychophysical theory of fidelity in time-sampled visual motion displays. J Opt Soc Am A 3:300-307. 